\title{
PERCEPCIONES SOBRE LA CORRESPONSABILIDAD ENTRE EL ESTADO ECUATORIANO Y LAS FAMILIAS EN EL CUIDADO DE LAS PERSONAS MAYORES
}

Patricia Celi Medina Investigadora predoctoral en Cátedra UNESCO, Mujeres, Desarrollo y Culturas y Grupo de Investigación en Sociedades, Políticas y Comunidades Inclusivas Universidad de Vic-Universidad Central de Cataluña patriciaestefania.celi@uvic.cat

RESUMEN: El presente artículo analiza las percepciones sobre la práctica de la corresponsabilidad entre Estado y familia, dispuesta por la Constitución ecuatoriana, para garantizar el bienestar de las personas mayores. Este objetivo se alcanzó mediante un estudio cualitativo con base en entrevistas semiestructuradas con personas responsables de la formulación y ejecución de políticas y servicios de cuidado para personas mayores, y con personas cuidadoras de sus parientes mayores. El estudio fue abordado a través del acercamiento teórico en torno a los orígenes de las desigualdades sociales y de género que se derivan de la organización social del cuidado y del desarrollo de los regímenes de bienestar en América Latina. Los resultados indican que existe un imaginario generalizado, asociado a la deuda moral en torno al cuidado y a las supuestas características innatas de las mujeres, que localiza a la familia como un sistema natural de cuidados. Mientras tanto, el rol del Estado se reduce a la protección social básica de las personas mayores en situación de exclusión social. Para las familias, se normaliza entonces como una realidad 
ineludible, una función pública débil en la socialización de los cuidados. Por su parte, desde el Estado, se sobreestima la capacidad de cuidado no remunerado de las familias, negando e invisibilizando sus demandas.

PALABRAS ClaVE: persona mayor; cuidados; corresponsabilidad; personas cuidadoras familiares; política social de cuidados ecuatoriana.

AвSTRACT: The present article analyzes perceptions about the practice of co-responsibility between the State and the family, as established in the Ecuadorian Constitution, to guarantee the well-being of the elderly. To do so the article uses a qualitative study based on semistructured interviews with people responsible for the formulation and implementation of policies and care services for the elderly, and with caregivers of their elderly relatives. The study was based on a theoretical approach to the origins of social and gender inequalities that derive from the social organization of care and from the development of welfare regimes in Latin America. The results indicate that there is a generalized understanding which locates the family as a natural system of care and which is associated with the moral debt around care and the supposed innate characteristics of women. Meanwhile, the role of the State is reduced to the basic social protection of the elderly in a situation of social exclusion. For families, the weak role of the State in the socialization of care then becomes normalized as an inescapable reality. For its part, the State overestimates the unpaid care capacity of families and denies and makes invisible their demands.

KEYWORDS: elderly; caregiving; co-responsibility; family caregivers; Ecuadorian social care policy. 


\section{Introducción}

En el Ecuador, a partir de la Constitución del 2008, se determinó que la población mayor constituye un grupo de atención prioritaria ${ }^{1}$. En los arts. 37 y 38 se describen, respectivamente, los derechos que el Estado garantizará a las personas de edad avanzada y las medidas que tomará para asegurar su cumplimiento. Específicamente, el art. 38 indica que, para garantizar los derechos de las personas mayores, el Estado «establecerá políticas públicas y programas de atención a las personas adultas mayores [...] y fomentará el mayor grado posible de autonomía personal y participación en la definición de estas políticas» (Asamblea Constituyente del Ecuador, 2008).

Según los principales instrumentos que, desde la instauración de la Constitución del 2008, orientan la política pública ecuatoriana, el aseguramiento de la plena vigencia de los derechos de los diferentes grupos de atención prioritaria, incluyendo las personas mayores, adquiere un principio de corresponsabilidad entre diferentes actores del país: Estado, sociedad y familias. Así, en el Plan Nacional para el Buen Vivir 2009-2013, documento con las herramientas de acción de la política social ecuatoriana, se ha señalado que se garantizará la promoción de «la corresponsabilidad pública, familiar y comunitaria en el cuidado de niñas, niños, adolescentes y personas dependientes» (Secretaría Nacional de Planificación y Desarrollo, 2009: 146). En el Plan Nacional para el Buen Vivir 2013-2017 se enfatiza la necesidad concreta de «fortalecer los sistemas de cuidado y atención integral a niños, niñas, adolescentes y adultos mayores en todo el territorio nacional, e impulsar la corresponsabilidad de la sociedad» (Secretaría Nacional de Planificación y Desarrollo, 2013: 119). Posteriormente, en el Plan Nacional de Desarrollo 2017-2021 se declara que en la actual directriz de la política pública ecuatoriana «se verán avances para alcanzar la corresponsabilidad en el trabajo del cuidado y la sostenibilidad de la vida; algo que creará oportunidades para la autonomía y la

\footnotetext{
I De acuerdo con el art. 35 de la Constitución ecuatoriana, las personas mayores conforman parte de los grupos de atención prioritaria: «Las personas adultas mayores, niñas, niños y adolescentes, mujeres embarazadas, personas con discapacidad, personas privadas de libertad y quienes adolezcan de enfermedades catastróficas o de alta complejidad, recibirán atención prioritaria y especializada en los ámbitos público y privado [...]» (Asamblea Constituyente del Ecuador, 2008).
} 
participación de las mujeres en otras esferas» (Secretaría Nacional de Planificación y Desarrollo, 2017:32).

Como lo evidencia el discurso expuesto en la hoja de ruta de las Administraciones de turno del Estado ecuatoriano, desde la creación de la última Constitución en el 2008, se ha desarrollado una amplia declaración de intenciones que ha profundizado en «lo imprescindible del principio de corresponsabilidad» en la garantía de bienestar de los distintos grupos de atención prioritaria, en el horizonte de elevar el «protagonismo» de la sociedad en este trabajo colectivo. No obstante, la evidencia en torno a la forma concreta en la que el principio de corresponsabilidad se traduce en una práctica cotidiana que comienza en la formulación de políticas desde las instituciones y alcanza el trabajo de cuidado no remunerado de las familias ha sido difícilmente evidenciada desde los ámbitos institucionales del Estado.

Alrededor de ello, una literatura específica documenta los desencuentros, en el contexto latinoamericano, entre las políticas públicas orientadas a personas mayores y la situación de las familias que se encargan de su cuidado. Desde varios estudios se ha demostrado cómo en ciertos países de América Latina, en los que se incluye Ecuador, existe un gran peso de las prácticas de producción informales. La mayoría de la población depende solo de arreglos familiares y comunitarios que subsisten en medio de mercados laborales y políticas públicas excluyentes. En particular, la familia es el actor por antonomasia en la oferta de los cuidados que se les brindan a las personas mayores. De esta forma, el ámbito doméstico se convierte en central porque transforma a los hogares en unidades productivas, a pesar de la falta de garantía de protección pública, que se compensa a través de herramientas como el trabajo informal, la emigración y las remesas (Huenchuan y Guzmán, 2007; Martínez, 2007; Del Valle, A., 2013; Acosta, E., 2015; Acosta, Picasso y Perrotta, 2017). Simultáneamente, otros análisis académicos sobre las familias como destinatarias de políticas públicas en América Latina han concluido que existen muy pocos canales de consulta a las familias y sus miembros, previos a la formulación de las políticas y el diseño de los programas. Son escasos los ejemplos de seguimiento para conocer cómo las familias responden a las 
políticas públicas y cómo asimilan los programas gubernamentales (Anderson, 2007; García y De Oliveira, 2011; Rossel, 2013). En general, estos estudios demuestran que, en la región, los criterios institucionales aprecian a la familia como un mecanismo natural de prestación de cuidados y bienestar para personas dependientes, pero, en el momento de diseñar políticas de atención a estos grupos poblacionales, el rol no remunerado de cuidado que otorgan las unidades familiares pasa a un plano secundario.

En torno a este escenario, en el que es cada vez más palpable la asincronía entre las políticas públicas y el papel de las familias a la hora de proveer cuidados a personas mayores, cabe preguntarse cómo interactúan los servicios de cuidado destinados a personas mayores implementados por el Estado ecuatoriano y la propia agenda de las familias que cuidan a sus parientes mayores, en el horizonte de garantizar la corresponsabilidad en el cuidado a este grupo etario. A raíz de este interrogante, el objetivo de este artículo es conocer las percepciones, tanto de las personas al cuidado de sus parientes mayores como de los actores que participan en los espacios de formulación y gestión pública en el Estado ecuatoriano, acerca del aporte de la implementación del principio de corresponsabilidad entre Estado y familias en la organización social del cuidado de las personas mayores. Alrededor de esto, la premisa con la que partió la investigación es que existe una idea generalizada de que la familia y el Estado proporcionan recursos complementarios para la atención de personas mayores; sostenida, sin embargo, en un imaginario social, conveniente en particular para el Estado, que asume prácticamente sin cuestionar que la familia, y dentro de ella, sus integrantes mujeres, es la responsable primordial del cuidado de sus parientes mayores.

Para responder a la pregunta de investigación, se aplicó una metodología centrada en el análisis cualitativo, utilizando como técnica de producción de información entrevistas semiestructuradas en profundidad a personas que integran instituciones públicas que diseñan y ejecutan políticas de atención a personas mayores y a personas que cuidan en sus unidades familiares a uno o varios parientes mayores. 
El texto ha sido dividido en cuatro apartados. En el primero se presenta el desarrollo metodológico del trabajo. En el segundo se plantea un acercamiento teórico en torno a la organización social del cuidado en América Latina y los orígenes de las desigualdades sociales y de género que se encarnan en esta. En el tercero, se discuten los argumentos que se utilizan desde la institucionalidad pública para defender la distribución de roles entre familia y Estado en torno al cuidado de las personas mayores. Y en el cuarto se examinan los discursos que preponderan en las personas cuidadoras familiares en torno a su rol en la garantía del bienestar de las personas mayores y el nivel en el que el Estado debería implicarse en este ámbito.

\section{Metodología}

La investigación ha sido de carácter eminentemente cualitativo y ha consistido en la realización de 27 entrevistas semiestructuradas: 19 a personas proveedoras de cuidados a sus parientes mayores y 8 a personas responsables, dentro de la Administración pública, de programas y servicios relacionados con los cuidados a personas mayores. En concreto, se entrevistó a personas que trabajan en el Ministerio de Inclusión Económica y Social (MIES), el Consejo Nacional de Igualdad Intergeneracional (CNII) y el Consejo de Protección de Derechos del Distrito Metropolitano de Quito (CPD-DMQ).

Se decidió emplear entrevistas como técnica de recolección de información dado que estas, según Carmona, Siavil y Ribot de Flores, «se producen en un contexto de interacción directo, personalizado, flexible y espontáneo, en el que se busca conocer motivaciones profundas y se obtiene respuestas libres, afectivas y comprometidas» (2007: 13). Por tanto, esta técnica facilitó en este estudio una aproximación profunda a los significados e interpretaciones que las personas dan al ejercicio de la corresponsabilidad en la organización social de los cuidados entre el Estado y las familias.

En la elaboración del muestreo de perfiles de personas proveedoras de cuidados de personas mayores, se tomó en cuenta la importancia de recoger la singularidad de situaciones de cuidado en la multiplicidad de 
sus dimensiones: en términos de las variedades de familias y formas de convivencias (se incluyeron familias extensas, nucleares, y de convivencia de solo abuelos-nietos, madres e hijo o padre e hijo), dando visibilidad a las diferentes condiciones en las que se proveen cuidados y a los diversos escenarios de relación de cuidados entre personas proveedoras y receptoras que se producen en el interior de las familias. Aun así, cabe aclarar que el control sobre el sexo de las personas entrevistadas en los ámbitos familiares fue limitado, por lo cual la mayoría son mujeres. Esto no responde a una decisión voluntaria de la investigadora, sino que refleja el universo feminizado en la organización social de los cuidados de personas mayores. Asimismo, cabe notar que, a nivel territorial, aunque hubiese sido deseable una representatividad a nivel nacional de la muestra de personas entrevistadas, los recursos disponibles de la investigadora permitieron concentrarla a nivel de la ciudad de Quito. No obstante, se tuvo la voluntad de incorporar perfiles que residen tanto el sector urbano como en el semiurbano de la ciudad, a fin de ampliar las miradas y percepciones sobre acceso a servicios de cuidados en dichas zonas.

Las entrevistas se realizaron entre mayo y agosto de 2016. Se aplicó un formulario de consentimiento informado, y con el fin de salvaguardar la protección de los datos de las personas entrevistadas, se ha otorgado a cada una un nombre ficticio.

\section{El Estado y las familias en la organización social de los cuidados: una revisión teórica}

Sofía, una de las mujeres cuidadoras que colaboró en este trabajo, tiene 64 años. Según la Constitución ecuatoriana, a ella le falta solamente un año para convertirse en una persona mayor. A diferencia de otras personas en las que físicamente la llegada de la vejez se advierte de modo progresivo, en ella el paso de los años parece haber llegado de golpe, sobre todo porque su estado físico se ha visto agravado por las secuelas que implican en su cuerpo las tareas del cuidado de su papá, Eduardo, que tiene 89 años. Durante la entrevista, Sofía contó lo siguiente: «Tengo artrosis, y, sin embargo, así le cuido. Pero cuando estoy mal si me hace sufrir, yo 
lloro y todo porque no le avanzo. A veces la enfermedad me hace sentir cansada, porque cuando se me inflaman las articulaciones yo me canso» (Sofía, 3 de julio de 2016). Cuando la enfermedad de Sofía se complica, ella descansa y su esposo o hijo intervienen para cuidar a Eduardo. En estos mismos momentos, ellos la atienden y acompañan a los servicios médicos que requiere. Como es factible notar aquí, la frontera que separa a la entrevistada entre su papel como cuidadora y su condición de receptora de cuidado es sumamente tenue. Su caso ilustra la complejidad de la definición estática de dependencia y autonomía en una relación de emisión y recepción de cuidados.

En efecto, es imposible clasificar a una persona como dependiente o independiente por su edad, y por su capacidad y predisposición para otorgar cuidado a los demás. Bajo esta premisa, recibir cuidado tampoco tendría que oponerse a la independencia o realización personal. Se trata, pues, de concebir el cuidado como parte de los vínculos cotidianos y asumir la vulnerabilidad y la dependencia de todas y todos como elementos constitutivos de la existencia y experiencia humanas (Pérez y López, 2011). El cuidado deja entonces de ser percibido como una actividad unidireccional, donde un cuidador «activo» independiente hace algo por un receptor "pasivo» dependiente y se presenta como una actividad que necesita de relaciones entre más de dos individuos. El cuidado es crucial para la vida humana porque la dependencia y la vulnerabilidad no son condiciones accidentadas que les llegan a los «otros». Son rasgos de todo el mundo que aparecen en distintas etapas del ciclo vital, y los más favorecidos pueden atenuar o negar su intensidad (Cerri y Alamillo-Martínez, 2012). Por lo cual, entender que no es la concepción dualista e inflexible sobre cuidador autónomo y receptor de cuidados dependiente sino la interdependencia lo que caracteriza las mutuas y constantes relaciones de cuidados de las personas permite ampliar la mirada sobre las esferas en las que se sitúan el trabajo de cuidado, así como los actores que intervienen en este.

En esa línea, el concepto «organización social de los cuidados», que es una adaptación regional, surgida en América Latina, del concepto social care elaborado por Daly y Lewis (2000), permite dar cuenta de las necesi- 
dades de cuidado que existen en un contexto determinado y cómo diferentes actores sociales responden a ellas, se combinan para esta provisión, y también del protagonismo que asume cada uno de ellos (Gonzálvez, 2016). Arriagada menciona que el concepto se refiere a las «interrelaciones entre las políticas económicas y sociales del cuidado. Se trata de la forma de distribuir, entender y gestionar la necesidad de cuidado que sustentan el funcionamiento del sistema económico y de la política social» (2010: 59 ).

La noción de organización social del cuidado se emparenta con la de «diamante de cuidado» (Razavi, 2007) como representación de la arquitectura que posibilita la provisión de cuidado. El diamante de cuidado indica la estructura a través de la cual se proporciona atención e incluye al Estado, las familias, el mercado y la sociedad civil. Aunque hay importantes diferencias entre estos ámbitos, las relaciones que se establecen entre ellos pueden producir su superposición. Por tanto, la idea de diamante de cuidado implica entender que las cuatro instituciones involucradas en el aprovisionamiento de los cuidados interactúan de maneras complejas, que las fronteras entre ellas no son nítidas ni inamovibles y que su trabajo no se produce de manera aislada (ibid.).

Rodríguez (2015) señala que la evidencia existente demuestra que la organización social del cuidado, en su conformación actual en América Latina en general, es injusta, porque las responsabilidades de cuidado se encuentran desigualmente distribuidas en dos escalas diferentes. Por un lado, hay una distribución desigual de las responsabilidades de cuidado entre hogares, Estado, mercado y organizaciones comunitarias. Y, por otro, la desigualdad en la distribución de roles también está presente entre hombres y mujeres. En síntesis, la evidencia muestra que el trabajo de cuidado es asumido mayormente por mujeres, concentrándose particularmente dentro de los hogares (Rodríguez, 2015; Nieves y Robles, 2017; Aranco et alii, 2018).

Varios factores son los que concurren simultáneamente en esta distribución dispar. El primero tiene que ver con la división sexual del trabajo. El trabajo de cuidado cumple una función esencial en las economías capitalistas: la reproducción de la fuerza de trabajo. Sin esta labor cotidiana 
que le permite al sistema reproducirse, el capital no podría disponer permanentemente de trabajadores y trabajadoras que han sido acondicionados y dispuestos a emplearse gracias al trabajo de doméstico no remunerado de las mujeres en el espacio privado de sus familias (Esquivel, 2011; Federici, 2018; D’Alessandro, 2018). Consecuentemente, la familia, «que es una construcción ideológica, se configura como una institución básica a la que se atribuye la responsabilidad del cuidado de sus miembros. Este hecho, que oculta la dimensión social del cuidado, resulta funcional para la reproducción de una sociedad desigual» (Comas d'Argemir, 2014: 1).

Es así como la división del trabajo que se consagra en el desarrollo del capitalismo y la separación entre familia y trabajo ha llevado a la vinculación automática de cuidado con las mujeres y la producción de mercancías con los hombres (Carrasco, Borderías y Torns, 2019). Efectivamente, en el capitalismo, las empresas se ahorran los costes de producción de la vida humana en aquella parte que corresponde a la población que no se halla en una situación económicamente ocupable. En cuanto al Estado, solo ha de asumir parcial y limitadamente las tareas de cuidado, presentándolas como si fuesen una actividad privada o propia del ámbito íntimo. Esta condición coloca a las mujeres en posición de aportar un trabajo imprescindible para la comunidad de forma no remunerada, como si fuera cuestión de voluntad individual, y, por tanto, una actividad privada respecto de la que el Estado, supuestamente respetuoso de la autonomía de los ciudadanos, no debiera intervenir (Izquierdo, 2004).

El segundo factor se refiere a la vinculación de las mujeres con el cuidado y la asistencia considerada intrínseca a su propia existencia como personas, lo cual desencadena que la familia constituya, a su vez, el marco natural en el que se ejerce el trabajo de cuidado. Esta vinculación descansa en los argumentos que, por un lado, llevan a concebir los cuidados mediados por los sentimientos y a las mujeres, como guardianas de esos afectos (Esteban, 2011). Y, por otra parte, en los argumentos defienden la obligación moral como la guía de la ética del cuidado, que es esa especie de deuda entre el flujo de cuidados de padres a hijos y a la inversa, que no se paga de forma inmediata, sino que está diferida en el tiempo (Comas d'Argemir, 2014). 
En esta línea, Esteban defiende la importancia de «desenmascarar esa asociación que se establece entre emociones y amor, por un lado, y asignación a las mujeres del trabajo de cuidar, basada en el hecho de que sean consideradas seres emocionales en mayor medida que los hombres» (2011: 70). Por su parte, Comas D’Argemir (2014) reclama que el énfasis en la dimensión moral tiende a idealizar el trabajo de cuidado y esconde la existencia de tensiones y conflictos, como los que se presentan en los cuidados de larga duración. Nos debemos preguntar, pues, «hasta qué punto se elige cuidar, ya que las elecciones están constreñidas por la estructura económica, por los patrones de género e incluso por las políticas públicas».

El tercer factor implica entender el cuidado como «una experiencia socialmente estratificada» (Benería, 2007; Marco y Rodríguez, 2010; Rodríguez, C. , 2015; D’Alessandro, 2018). En efecto, las familias que pertenecen a diferentes niveles socioeconómicos cuentan con distintos grados de libertad para tomar decisiones sobre la forma de organizar el cuidado de sus miembros. Tal cual lo explica Rodríguez (2015), las mujeres que viven en hogares con ingresos medios o altos tienen la posibilidad de adquirir servicios de cuidados en el mercado o de pagar por el trabajo de cuidado de otra mujer. Esto alivia la presión sobre su propio tiempo de trabajo de cuidado no remunerado, liberándolo para otras actividades. Estas opciones se encuentran limitadas o directamente no existen para la enorme mayoría de mujeres que viven en hogares de estratos socioeconómicamente bajos. En estos casos, no existen oportunidades para reducir la presión sobre el tiempo de trabajo no remunerado. La participación económica se ve restringida o se produce en medio de una tensión por la carga de trabajo remunerado y no remunerado acumulada. De este modo, la forma en la que sucede la organización social del cuidado puede resultar en sí misma un factor de reproducción y profundización de la desigualdad.

El cuarto factor tiene que ver con la forma que adopta la organización social del cuidado a la luz de los recorridos históricos de los regímenes de bienestar. Ya en el siglo xx, en la génesis de los estados de bienestar, se produce el traspaso de competencias entendidas como privadas a la in- 
stitución pública (Carbonell, Gálvez y Rodríguez, 2014; Lewis, 2019). Sin embargo, hoy por hoy, la familia sigue realizando una serie de funciones que aún no han sido corresponsabilizadas por el Estado en la misma dimensión que otras responsabilidades. Así, cuestiones como el cuidado han sido consideradas como unas de las tantas funciones y servicios dispuestos por las familias, y dentro de ellas, en este caso, por sus integrantes mujeres. De esta manera, la participación del Estado ha quedado reservada para aspectos muy puntuales o como apoyo de los hogares cuando las situaciones particulares lo ameritan (Eguren, 2001; Martínez y Voorend, 2009; Batthyány, 2015; Vega, Martínez y Paredes, 2018).

América Latina, en particular, ha enfrentado de distintas formas y en distintos momentos históricos el «dilema del cuidado» (O'Connor, 1996) en la distribución de entrega de bienestar y protección social a los grupos poblacionales. O expresado en otras palabras, en la región es casi permanente el dilema acerca de los criterios con los que se reparten el peso e importancia entre las esferas de producción de bienestar: Estado, mercado, familias y comunidad en la provisión de cuidado. Es así como la articulación de estas ha dado lugar a la existencia de distintos regímenes de bienestar que originan diversas tipologías (Wood y Gough, 2006; Martínez, 2007; Antía, 2018).

Debido a eso, Martínez (2008) encuentra que los regímenes de bienestar deben ser comprendidos como constelaciones en las que se ejercen distintas prácticas que dirigen y asignan los recursos. Por ello, estos regímenes pueden incluir estados de bienestar en su garantía mínima adecuada de este principio. En consecuencia, el estudio de los regímenes de bienestar tendría que ser abordado desde la distribución de los recursos, sea esta o no llevada a cabo por el Estado. La autora recupera entonces el concepto de familiarización de Orllof (1993), el cual da cuenta de la disponibilidad de trabajo femenino no remunerado en la división social del trabajo para el análisis de los regímenes de bienestar en América Latina. Ella menciona que la familiarización permite incorporar el género como una dimensión central del análisis de los regímenes de bienestar, en tanto las prácticas de asignación de recursos se organizan en torno a la división sexual del trabajo (Martínez, 2008). Esta dimensión es cru- 
cial entonces en el análisis de los regímenes de bienestar de una región como la latinoamericana, donde las esferas familiar y comunitaria han sido históricamente protagonistas en la provisión de bienestar y cuidados (Aguirre, 2007; Martínez, 2008; Gherardi, Pautassi y Zibecchi, 2012; Minteguiaga y Ubasart-González, 2013; Rico, 2016; Ascensio, 2017).

En esa línea, el concepto de (des)familiarización alude a eximir, en algún grado, la responsabilidad del cuidado y de provisión de bienestar como exclusiva responsabilidad privada y femenina (Orloff, 1993). Sobre este concepto, Esping-Andersen (2000) aclara que la desfamiliarización no implica una connotación «antifamiliar», sino que más bien tiene que ver con los mecanismos que permiten relativizar o reducir las responsabilidades ligadas al bienestar y los cuidados de las familias, a través de la acción del Estado, mercado o comunidad como agentes activos en la protección social y generación de bienestar. Por tanto, el análisis de los niveles de desfamiliarización de las políticas sociales acercaría «al estudio de la provisión de bienestar y cuidados que permiten la reproducción de la vida, analizando así los actores sobre los que recae esta, sus formas, sus intensidades, e inclusive las actividades asociadas, entre otros» (Minteguiaga y Ubasart-González, 2013: 47).

Con esto en mente, en las siguientes páginas se explora la distribución desigual de roles de los trabajos de cuidados, entre Estado y familia y entre hombres y mujeres dentro de los hogares, en la práctica corresponsable del aseguramiento de bienestar para la población mayor. La literatura ha permitido advertir que, en ciertos regímenes de bienestar, el cuidado no es visto como un derecho a lo largo del ciclo vital en el que todos nos vemos involucrados de formas distintas (ya sea como receptores o proveedores de este) y que se puede intensificar en ciertas edades, como la vejez. Para muchos actores sociales como los Estados, la provisión de bienestar a las personas mayores a través de los cuidados es entendida como una labor propia de ciertas esferas, como la familia, cuya ausencia es la única razón para brindar ciertas asistencias. Esta condición para la intervención pública poco o nada representa la diversidad de condiciones en las que se envejece y en las que se necesitan atenciones específicas. 


\section{Las familias vistas desde el Estado como cuidadoras por naturaleza}

Los cuatro factores generadores de un desigual reparto del trabajo de cuidados entre los diferentes actores sociales que integran la red de la organización social de los cuidados, explicados en la sección anterior, han concurrido simultáneamente en el trayecto que ha seguido el Estado ecuatoriano en su conformación como régimen de bienestar. Martínez (2007) describe el régimen de bienestar de Ecuador como uno que combina un discurso individual y otro mercantil del bienestar, con un pasado no tan lejano marcado por fuertes expectativas con el manejo colectivo de riesgos. En el país, desde enero del 2007, con la llegada de Rafael Correa a la presidencia de la República, se buscó revertir las condiciones materiales y simbólicas heredadas de la implementación de más de dos décadas de políticas neoliberales derivadas del Consenso de Washington (Minteguiaga y Ubasart-González, 2013). El Gobierno de Correa pretendió «desmontar el núcleo duro de la agenda neoliberal y recuperar ciertas funciones de bienestar social y regulación económica, como se venía realizando, aunque de modo muy desigual, en los años previos al ajuste estructural» (Ramírez, 2006, citado por Minteguiaga y UbasartGonzález, 2013: 15). Con el propósito de vencer la crisis de la «hegemonía neoliberal», Correa llevó adelante la elaboración, sanción y aprobación popular de un nuevo texto constitucional que, a la par de la instrumentalización de políticas y leyes, pretendía, «bajo un discurso de representación del interés general y con vocación universalista», un «retorno del Estado como campo de las decisiones colectivas y un retraslado de la acción política desde el ámbito social/mercantil hacia la interfaz estatal/ pública» (Minteguiaga y Ubasart-González, 2013: 16).

Estas dinámicas de transformación estatal procuraron verse reflejadas, entre otras cosas, principalmente, en la fuerte inversión social y la extensión de políticas del bienestar, en especial, aquellas que han sido incorporadas para satisfacer las demandas de la población de atención prioritaria. En 2017, cuando Lenín Moreno asumió la presidencia de la República, el nuevo Gobierno que se pensó inicialmente como la continuación del proyecto político de su antecesor, al poco tiempo de ser inves- 
tido, comenzó a marcar distancia. Como resultado, desde el Estado se armó un entramado institucional y legal para sobrevivir y cerrar la pienza neoliberal que ya se venía sembrando con las últimas medidas económicas salvavidas de Rafael Correa. Un discurso de crisis autoinducida llevó al Gobierno a enarbolar políticas de ajuste sociales sostenidas y medidas de austeridad parar lograr la estabilidad y equilibrio fiscal, reestructuración y optimización del Estado, equilibrio externo, sostenibilidad de la dolarización y reactivación productiva (Secretaría General de Comunicación de la Presidencia, 2018). Esta claudicación neoliberal vino acompañada, sin embargo, de una aparente apuesta dirigida a que el Estado asumiese sus tres obligaciones básicas: «respetar, proteger y realizar», como garante de derechos a lo largo del ciclo de vida (Secretaría Nacional de Planificación y Desarrollo, 2017). Con el paso del tiempo, ciertos ámbitos de este horizonte, especialmente los que tienen que ver con derechos laborales, educativos y de salud, han cedido a los recortes sociales, mientras que otros, como la seguridad y protección social, se mantienen en la cuerda floja, en un intento por no desarticular el proyecto social en la defensa de los derechos de los «más vulnerables» que ha caracterizado la carrera política de Moreno.

Consecuentemente, en la última década, con el objetivo de sostener los rezagos de la reapropiación de recursos y el restablecimiento de la capacidad de planificación del Estado ecuatoriano en el ámbito social, las instituciones públicas encargadas del bienestar de los diferentes grupos de atención prioritaria, como las personas mayores, han incorporado un conjunto de discursos sobre la construcción de políticas estructurales de bienestar y el aumento de cobertura y la calidad de servicios públicos orientados a los cuidados, a fin de justificar la forma en la que se hacen efectivos los derechos sociales de este y otros conjuntos poblacionales.

Esos discursos dejan ver el modo en el que, desde las instituciones encargadas de formular, diseñar y ejecutar política pública, se entienden los roles de la provisión de cuidados para las personas de edad avanzada, y, al mismo tiempo, son indicadores de cómo funciona el principio de corresponsabilidad en el ejercicio de estas funciones. 
Sobre este principio, Isabela, responsable de la Secretaría Ejecutiva del CPD-DMQ en el 2016, explica que la corresponsabilidad apunta a procurar el involucramiento de la sociedad en los procesos de desarrollo y cuidado de las personas, cuyos derechos son garantizados por el Estado. Al respecto menciona:

Además, nosotros sabemos, y lo dice la Constitución, que la familia es la principal responsable del cuidado y protección de sus miembros, de la vida y del desarrollo. A falta de familia, el Estado asume esa responsabilidad. Pero mientras exista la familia, esta es la responsable directa. Y la sociedad también está para dar sentido a la corresponsabilidad, fortaleciendo y apoyando al trabajo de la familia (Isabela, 15 de julio de 2016).

Este testimonio resume las representaciones que todas las personas representantes de las instituciones del Estado que colaboraron en este estudio mantienen sobre el rol del Estado y la familia frente al cuidado de la población de edad avanzada. Con base en la norma constitucional, se percibe al Estado como la esfera macro que, a través de la formulación de políticas públicas, de la provisión de servicios básicos, de la generación de marcos legales, etc., garantizará el bienestar de la población mayor, sobre todo de aquella que se encuentra en un contexto de vulnerabilidad social, en el cumplimiento de sus derechos mínimos ${ }^{2}$. El tema de brindar cuidados de manera generalizada recae de forma fundamental y automática en el ámbito privado de la familia.

\footnotetext{
2 El MIES, institución rectora de las políticas sociales para personas mayores en Ecuador, tiene una cartera de servicios destinados al cuidado y bienestar de este grupo poblacional. Estos se sostienen en dos políticas clave. (1) La primera es la política de aseguramiento no contributivo, que tiene por objeto cubrir a los grupos que se encuentran en situación de pobreza y vulnerabilidad frente a la necesidad de ingresos, con el fin de evitar el deterioro del consumo básico. (2) La segunda política pretende «garantizar la atención especializada durante el ciclo de vida a personas y grupos de atención prioritaria, en todo el territorio nacional, con corresponsabilidad entre el Estado, la sociedad y la familia» (2016: 23). Para esto, el MIES ha establecido los siguientes servicios y prestaciones que se destinan únicamente a las personas que viven bajo la línea de la pobreza o que no cuentan con un referente familiar (MIES, 2020): a) centros gerontológicos de atención directa: infraestructura gerontológica construida directamente por el MIES o intervenida por este -en total existen 14 centros de atención directa a nivel nacional, con una cobertura aproximada de 70881 personas-, y b) convenios para cuidado y atención de personas adultas mayores - el MIES ha suscrito un total de 428 convenios con instituciones privadas en todo el país para atender a personas adultas mayores a través de diversas modalidades de atención-.
} 
Según estas autoridades, existen varias razones para considerar esta separación de funciones. La causa primordial se encuentra en la Constitución, la cual, aunque no señala explícitamente que la familia es la proveedora directa de cuidados, sí alude a esto cuando en el art. 38 menciona que «la ley sancionará el abandono de las personas adultas mayores por parte de sus familiares o las instituciones establecidas para su protección» (Asamblea Constituyente del Ecuador, 2008). Las otras razones se sostienen en los argumentos mencionados anteriormente en el apartado teórico de este artículo, que tienen que ver con la percepción que conecta automáticamente a las mujeres como fuente inagotable de afectos que son, difícilmente intercambiables y motor principal de un «buen cuidado», con el cuidado visto como un compromiso que los parientes más jóvenes deben saldar con las personas mayores como un mecanismo de reconocimiento y gratitud. Por eso, en las instancias del Estado, se percibe la familia como una institución que cuida tanto por su naturaleza como por la deuda moral que se concibe dentro de sí, generación tras generación.

En concreto, al referirse sobre cómo influyen las políticas públicas en el nivel de implicación que tienen las familias en el trabajo de cuidados, Armando, responsable en 2016 de la Secretaría Técnica del CNII, advierte el papel central de la familia atendiendo a consideraciones más pragmáticas sobre la no suficiencia de los recursos que están a disposición del Estado: «En el Ecuador, por el gasto público que tiene, que está muy lejos de otros países que pudieran tener sistemas de cuidado más robustos, se duda de la viabilidad de que el Estado se pueda hacer cargo del cuidado, es decir, de incrementar centros gerontológicos y pagar a las personas que trabajen en ellos» (Armando, 10 de junio de 2016). Bajo este criterio, la percepción de las familias como dadoras naturales de cuidados se refuerza, además, por argumentos utilitarios que enfatizan en lo primordial de la optimización y ahorro de recursos del Estado. En este sentido, las familias, al cuidar a sus parientes mayores, no solo estarán cumpliendo su obligación moral con ellos, sino también una deuda social con el Estado, y prácticamente impuesta por este. 
Armando menciona que el hecho de que las familias no respondan adecuadamente a esa «responsabilidad deseada desde instancias institucionales antes que colectivas» deviene, sin embargo, en una sanción que es más bien social: «No es deseable que el Estado se haga cargo del cuidado, porque la persona no debería morir aislada lejos del seno en el que compartió y sustentó en otras etapas de la vida, salvo el caso en el que no tengan referente familiar. Porque al final la privación del medio familiar podría ser una de las situaciones que hacen muy traumática la vida de los adultos mayores» (Armando, 10 de junio de 2016).

El punto de vista de Armando es el reflejo de la retórica en el desarrollo de la política de cuidados para personas mayores del MIES. Este ministerio es el ente rector de un conjunto de modalidades de atención ${ }^{3}$ para personas mayores, que están destinadas de forma exclusiva a atender las necesidades de cuidado de aquellos grupos poblacionales que viven bajo la línea de la pobreza o que no cuentan con un referente familiar. De esta forma, los servicios de cuidado no solo han sido absorbidos por una política más amplia de combate a la pobreza, sino que, además, se despliegan en un escenario en el que el Estado ha normalizado que la unidad familiar existe como el núcleo insustituible de cuidado a sus miembros, y, por tanto, los ámbitos institucionales públicos solo deberán intervenir cuando la familia se ausente.

Como consecuencia de lo anterior, a pesar de que el papel de la familia como cuidadora vital es constantemente recalcado por las personas entrevistadas de las instituciones del Estado, casi ninguna halla una respuesta concreta para dar cuenta del trabajo de este fortaleciendo capacidades y facilitando recursos y oportunidades a fin de que los integrantes familiares cuenten con mayores soportes para su labor en la provisión de

\footnotetext{
3 Todos los servicios de atención a personas adultas mayores, sean directos o por convenios, operan bajo un sistema de modalidad que puede ser de cuatro tipos. (1) Residencial: servicios de acogida para la atención y cuidado ofrecidos a personas adultas mayores que no pueden ser atendidos por sus familiares o que se encuentran en situación de abandono y que manifiesten su voluntad de ingresar. (2) Diurna: servicio de atención diaria que brinda una atención integral sin internamiento a personas adultas mayores con dependencia leve, intermedia o moderada. (3) De atención domiciliaria: servicio para personas adultas mayores que se encuentran en situación de dependencia o discapacidad temporal que les impide movilizarse fuera de su hogar. (4) De espacios alternativos diurnos: son espacios para el encuentro y socialización de las personas adultas mayores, donde se realizan actividades recreativas y de integración (MIES, 2016).
} 
cuidados y para que se reduzca, al menos, la carga de trabajo destinada en este sentido a las mujeres en el ámbito doméstico. Según Acosta, incorporar los cuidados como una responsabilidad social compartida produce beneficios generalizados. La consecuencia de ello son las políticas de cuidado o las políticas de conciliación con corresponsabilidad social, un paraguas que engloba acciones diversas que de una $u$ otra forma impactan en la distribución del trabajo no remunerado. Se trata de «acciones orientadas a compatibilizar la vida laboral, personal y familiar al tiempo que se busca proteger los derechos de quienes necesitan de cuidado» (2013:16). Así, dentro del esquema del diamante de cuidado, «el Estado es un actor muy importante en la distribución de roles y responsabilidades, por cuanto el peso que este tenga en la provisión de cuidado determinará la carga que se delega al resto de los proveedores de cuidados» (ibid.: 17). Empero, los testimonios de las personas entrevistadas reflejan la idea de una reducida intervención estatal, que, más allá de acciones directas con los titulares de derechos de las políticas de cuidados, es decir, las personas de edad avanzada, se ha mantenido al margen de las demandas de las personas cuidadoras familiares que los atienden.

En efecto, aunque se reconocen las dificultades siempre presentes en las tareas de cuidados destinadas a personas mayores dentro de las familias, no existe una política o instrumento contundente para simplificar estos problemas y apoyar el trabajo familiar de cuidado. Más bien, parece que se ha construido una sensación de idealización del trabajo familiar, que llega a considerar a los hogares como los portadores de la suficiente autonomía y capacidad para resolver cuestiones como la calidad de la atención a la persona mayor. Por ejemplo, Anabel, exfuncionaria de la Dirección de la Población Mayor del MIES, encuentra que si es la familia quien debe velar por la persona mayor, es esta la que debe buscar por sus propios medios los mecanismos para asegurar su bienestar. Por eso, explica:

Lo que necesitaría la familia es acercarse a las instituciones del Estado a pedir asesoramiento sobre cómo atender a sus adultos mayores con calidad y calidez. Si nosotros como hijos contratamos a una cuidadora y queremos asegurarnos de que su atención sea adecuada, yo como hija 
me acerco a ella y le digo que se vaya a una residencia gerontológica a capacitar y vea cómo es el cuidado al adulto mayor (20 de junio de 2016).

Este tipo de discurso es uno de los tantos argumentos que se identificó durante la realización de entrevistas, a fin de legitimar la concentración de responsabilidades de cuidado en el entorno familiar y el desentendimiento de las atribuciones del Estado en estas tareas. La idea de la familia autosuficiente es útil desde esta perspectiva, porque, por un lado, invisibiliza las implicaciones para las oportunidades de trabajo de las mujeres y su independencia económica cuando una porción importante del cuidado no puede delegarse a terceros y debe realizarse dentro del hogar, y, por otro, facilita la falta de reconocimiento del trabajo no remunerado dentro de las cuentas nacionales, lo que equivale a considerar como «no productoras», «inactivas», «no ocupadas» y fuera de ciclo económico a las personas que dedican su tiempo al cuidado de otros sin percibir por ello remuneración. Más allá de consideraciones de justicia en el reconocimiento de las contribuciones a la producción, hay que subrayar que la invisibilidad de esos aportes afecta adversamente a la justicia de la distribución de recursos, beneficios y compensaciones derivados de esa producción (Gómez, 2008).

Desde esta mirada, es imperante reflexionar sobre la forma en la que los hacedores de políticas sociales entienden la responsabilidad del Estado como garante de derechos de la población mayor. Si el bienestar de este grupo de atención prioritaria es visto solo desde la mirada de las personas receptoras de cuidado, y no desde quienes lo proveen, es evidente que el análisis de prioridades carece de un enfoque más integral que supere la visión del titular de derecho como un individuo aislado de interacciones con otros, que también tienen roles centrales en su bienestar.

El análisis cualitativo evidenciado en este apartado permite varias reflexiones sobre las percepciones en torno a la familiarización de la política de cuidados en Ecuador. En el país, la oferta de servicios de cuidado para personas mayores está instalada en un marco institucional de respuesta a las condiciones de pobreza y vulnerabilidad. No se responde a demandas estructurales de provisión y recepción de cuidados, sino que estas se insertan como uno de los tantos medios para resolver contextos 
agudos de exclusión social. Los testimonios de las personas funcionarias entrevistadas reflejan un enfoque de concepción de la política en el que está ausente una mirada de responsabilidad pública frente a unas necesidades de cuidado que desde el criterio de las personas entrevistadas encontrarán respuestas en los espacios familiares, y, en especial, en el trabajo de sus integrantes mujeres. Por esta razón, la oferta de cuidados solamente está presente para los sectores poblacionales que no cuentan con referente familiar. Este condicionamiento para el acceso, sumado a la alta focalización, demuestra cómo las políticas aparentemente fueron construidas con base en preceptos naturalizados sobre el rol central de las mujeres frente a los cuidados, y, por tanto, de la familia, como el principal espacio de afecto, y capacidades óptimas para cuidar. Desde el Estado no solo que se invisibiliza la importancia de redistribuir las responsabilidades de cuidado desde las familias a otras instituciones sociales, sino que tampoco se han propiciado políticas y programas para apoyar y sostener el trabajo en la provisión de cuidados de las unidades familiares.

\section{Cuando los cuidadores familiares no reclaman apoyo porque reafirman sus roles como ineludibles}

La asociación instantánea del cuerpo de las mujeres a un rol de cuidadoras, que ha resultado además en la naturalización del entorno familiar como el centro más propicio, fundamental y difícilmente irremplazable de provisión de cuidados, es uno de los principales obstáculos para vencer el contrato desigual de género en la organización social de los cuidados. Todos estos imaginarios no solo han tenido efectos en los roles que asume el Estado en la organización social de los cuidados, sino que también han ocasionado, como se presenta en esta sección, que dentro de las mismas familias se marquen ciertas expectativas, y percepciones - que ahondan la desigualdad social y de género que caracteriza a las redes de provisión y recepción de cuidados - frente a sus propias responsabilidades y a las que debería cumplir el Estado respecto al cuidado de las personas mayores. 
En el proceso de recolección de datos con las personas cuidadoras familiares, fue posible notar que la naturalización de su entorno como un espacio de provisión de cuidados no solo sale a flote cuando estas determinan las razones que las llevan a cuidar a su familiar mayor, sino, además, cuando establecen sus criterios y proyecciones sobre el rol del Estado. Los temas de salud y recreación alrededor de la población mayor fueron dos constantes en las respuestas sobre el papel del Estado en la garantía de sus derechos. Para las personas cuidadoras, a fin de garantizar el bienestar de las personas mayores, es urgente que el Estado mejore la calidad y calidez de la atención médica a las personas de edad avanzada y que, a la vez, incremente el número de espacios a los que estas pueden acudir para compartir con gente de su edad y ocupar su tiempo libre. El testimonio de Fabián advierte esta percepción:

Lo que necesita mi mamá y todos los adultos mayores es recursos físicos y profesionales en los hospitales, porque esta es la época de las enfermedades. Con los años aumentan las necesidades de cosas para la salud y también para que mi mamita se entretenga. Es muy difícil. El adulto mayor necesita recreación, por lo menos infraestructura para mejorar aspecto social, para entretenerse (13 de agosto de 2016).

Aunque, definitivamente, el acceso a sistemas de salud eficientes y a espacios recreacionales constituye un apoyo crucial a la labor de cuidado de las familias, estos factores son apreciados por las personas entrevistadas como mecanismos sustanciales que incrementan directamente la calidad de vida de las personas mayores. Los entrevistados no los vinculan a dispositivos que podrían posibilitarles redistribuir la carga de su trabajo de cuidado. En ese sentido, de primera mano, las personas entrevistadas señalan que el Estado ha hecho muy poco por la población mayor, dado que aún son escasos la infraestructura y los recursos de salud disponibles para aportar a su bienestar.

A la par, los testimonios en torno a la falta de recursos para cuidar con calidad dentro de casa y sobre la serie de inconvenientes que ha representado para las personas familiares el no saber cómo responder a diversas circunstancias del cuidado surgieron con regularidad. El caso de Cecilia, quien cuida de su mamá, Inés, es un reflejo de esta situación. Cuando 
Inés sufrió una fractura en su pierna derecha que la dejó impedida para caminar, el inicio de su etapa de dependencia representó un quiebre en la vida de Cecilia:

Cuando mamá salió de la clínica luego de la operación de su pierna, nadie se imaginó lo que sería al llevarla a la casa. Yo tenía una persona que la cuidaba de lunes a viernes, pero sábado y domingo me dejaron a mi solita. En ese tiempo era supercomplicado virarla a mi mamá. Yo ahí le llamaba al doctor y le decía qué hago con mi mamá, yo estoy solita. Yo no la podía mover y ella tenía que hacer rehabilitación. También había que hacerla rodar para cambiar la sábana. Yo ahora he aprendido mucho pero en ese entonces no sabía nada. Yo realmente me ponía a llorar (8 de junio de 2016).

Frente a esta situación, Cecilia optó por contratar a una persona que asista los fines de semana a hacer la rutina habitual de aseo a Inés. La entrevistada admite que sin la ayuda de cuidadoras remuneradas hubiese sido imposible atender de forma adecuada a su mamá, porque nunca tuvo una fuente de aprendizaje confiable sobre cómo proceder frente a ciertas tareas de cuidado.

Simultáneamente, otro grupo de personas entrevistadas también manifestaron no contar con la misma posibilidad de Cecilia para invertir recursos en cuidados profesionales externos. No obstante, señalaron que el afecto que solo se despliega en el ámbito familiar puede convertirse, entre tantas cosas, en un incentivo para sortear todo tipo de barreras en el trabajo de cuidados. Ese es el caso de Jacqueline. Ella recibe un sueldo por parte de todos sus hermanos a cambio de ejercer los trabajos de cuidados permanentes para su madre, que padece de párkinson. Jacqueline no cuenta con ninguna formación en cuidados a largo plazo, sin embargo, afirma: «Yo solita yendo al hospital, poco a poco de lo que me decían los doctores, con amor aprendí para poder ayudar a mi mamá [...]. Ella me dio la vida, y ahora tengo que cuidarla como ella me cuidó a mí. Nadie más la va a poder cuidar igual» (3 de julio de 2016). De manera similar, Cristina también destacó en su testimonio la trascendencia del afecto, como una característica sustancial de las familias que las convierte en espacios insustituibles del cuidado: 
A muchos ancianitos que están en los asilos les cuidan [...] pero si no les ven a sus hijos se van a sentir muy mal. Pueden tener la mejor atención, la mejor medicina, pero si no tienen afecto se van a sentir mal. Entonces la mejor medicina para cualquier adulto mayor tenga la enfermedad que tenga es estar con los hijos, es verse amado por todos (3 de julio de 2016).

Igualmente, fueron constantes los testimonios acerca de la imposición social de las familias para cuidar de sus parientes mayores, atendiendo a una deuda de cuidado con ellos, que no se puede sortear, porque moralmente deja en suspenso ese gesto de reciprocidad, y eso, para las familias, es un acto reprochable. Por ejemplo, Martha trasluce este escenario así: «Un abuelito o una abuelita es una persona que dio su vida por cuidar a sus hijos y a sus nietos, y lo mínimo que podemos hacer por ellos como familia es darles una vida digna, una vida en la que se respeten sus derechos, una vida en la que sean valorados, escuchados y atendidos» (Martha, 13 de junio de 2016).

Las experiencias de Jacqueline, Cristina y Martha evidencian que, por un lado, la idea de las familias vista como una fuente inagotable de amor y afecto, y, por otro, el imperativo moral en relación con saldar la deuda social con las personas mayores son percepciones que las personas proveedoras de cuidados han colocado como justificaciones para atribuir a los espacios familiares las características de óptimos y propicios para otorgar cuidado. Más allá de las inequidades de clase y género, las tensiones, conflictos, desazones, falta de capacidades y recursos, como los advertidos en el caso de Cecilia; la familia está abocada a cuidar por sobre todas las cosas. Por esa razón, ninguno de los entrevistados indicó su malestar o disconformidad con el hecho de que el Estado haya tomado una posición tan pasiva para responder a sus demandas como personas cuidadoras, ni mucho menos con la idea de que el mercado tampoco se ha presentado como una alternativa oportuna y accesible para solventar esas dificultades.

La noción de corresponsabilidad respecto al cuidado de las personas de edad avanzada surgió únicamente cuando se preguntó a los familiares sobre las responsabilidades que, en torno a los cuidados, podría o debería asumir el Estado. Nadie admitió como posibilidad centrar en su totalidad 
en las esferas públicas las demandas de cuidado de las personas mayores. De forma generalizada, se podía intuir poca confianza en las capacidades y voluntad del ámbito estatal para llevar adelante responsabilidades vinculadas al cuidado. Inclusive, esta percepción se vio fortalecida con el hecho de que nadie manifestó conocer sobre las modalidades de atención disponibles para personas mayores que cuentan con bajos niveles socioeconómicos ofrecidas por el MIES. El testimonio de Sonia ejemplifica estas percepciones:

Mis padres, gracias a Dios, no han necesitado beneficios del Estado. Ni siquiera sé que servicios tiene el Estado para los adultos mayores. Pero se ven muchos botados en las calles, aún. Se ven muchos adultos mayores que pasan cosas tristes y solos. Entonces no sé si se está abarcando todo lo que el Estado promociona. Yo todavía veo en las noticias que los adultos mayores mueren solos en sus casas porque no tienen quien los vea. No sé dónde están las políticas estatales. No entiendo cómo llegan adultos mayores a un hospital con un grado de desnutrición tan grande. ¿Dónde estaban las políticas estatales? (13 de junio de 2016).

Para un grupo de personas entrevistadas, la única alternativa de cuidado que no sea la familia es la ofrecida por los centros gerontológicos privados, que para la mayoría tampoco es considerada una opción plausible, no solo por su alto costo, sino también porque simbolizan todo lo contrario a lo que la familia puede suministrar: desatención, escasez de afecto, olvido, abandono, etc. El testimonio de Mercedes trasluce esta percepción sobre estos lugares de atención privada:

Cuando mi mamá sufrió la fractura de su pierna, con dos hermanos recorrimos centros de cuidado del adulto mayor y realmente son una calamidad. A nosotros se nos iban las lágrimas. Entras y esas casas huelen a orinas. Eran 12:30 del día y los viejitos estaban en el patio, con un chupete y una galleta. Les tienen completamente hacinados. No tienen habitaciones confortables, sino que están en medias aguas húmedas en la parte de atrás de las casas (8 de junio de 2016).

Más allá de cuestionar la veracidad de la afirmación de Mercedes, cabe mencionar que este tipo de imágenes son las que comúnmente se gener- 
alizan sobre la institucionalización del cuidado. Son este tipo de percepciones las que han conducido, sobre todo entre las mujeres cuidadoras y su entorno social, a considerar que es más favorable para la persona mayor que ellas renuncien a otras actividades, sacrifiquen tiempo, recursos y energías para dedicarse a cuidar. El cuidado afectuoso, que se posterga como una deuda intergeneracional en los círculos familiares, se convierte en un imperativo en vigilancia constante bajo la lupa de la sanción social. Estos elementos vistos por las personas como «compromisos con el cuidado» de sus parientes mayores conducirían a las familias a prescindir de otras alternativas de cuidado, incluso las ofrecidas por el mercado como opciones posibles para redistribuir los roles de cuidado. En esta línea es destacable que no solo la clase se concibe como una barrera para socializar las demandas de cuidado, sino que, sumados a ella, y como factores igual de cruciales, cuentan los imaginarios sobre los potenciales perjuicios y amenazas de otros ámbitos proveedores de cuidados, en la oferta tanto pública como privada. En este punto resulta llamativo que el escepticismo y la falta de confianza sobre las capacidades de cuidado en otras instancias, de ninguna forma, saltan a la luz en los testimonios de las personas entrevistadas al hablar del cuidado proporcionado en entornos familiares.

Por su parte, otro grupo de entrevistadas se reapropió del discurso oficial y destacó la factibilidad del Estado como proveedor de cuidados, pero únicamente cuando se trate de atender las necesidades de personas mayores en condiciones de pobreza, que no han podido trabajar para recibir una jubilación adecuada, que han envejecido en soledad y no pueden solventar su cuidado, o bien aquellas que han sido abandonadas por sus familiares. Sobre esto, César fue enfático al afirmar lo siguiente:

Supongamos que el adulto mayor no tiene familia, entonces el Estado debe hacerse cargo, pero mi papá, sí tiene quien responda por él, no puedo decirle al Estado que se haga cargo. También hay casos de familias que teniendo posibilidades le dejan abandonado al adulto mayor. Las leyes tienen que estar claras. Cuando el adulto mayor tiene familia, no le pueden botar. Si está solo, el Estado le debe cuidar, no tiene por qué estar en la calle mendigando (1 de julio de 2016). 
Así, las ideas sobre la incapacidad de ciertos actores, como el Estado o los centros geriátricos, para brindar cuidado de calidad, o sobre la necesaria focalización de acciones y programas entre la población mayor, reflejan el alcance de la interiorización del discurso que naturaliza al ámbito familiar como un espacio suficiente e idóneo para desarrollar las tareas de cuidado. La asimilación de estos roles como invariables e inflexibles ha conducido a la normalización de la distribución desigual de responsabilidades en la organización social del cuidado. Para el Estado es útil manejar un discurso de corresponsabilidad, por cuanto, en el horizonte de compartir roles con las familias, se ha legitimado la facultad, por un lado, de monopolizar en ellas las tareas para el aseguramiento del bienestar de la población mayor en alusión a las destrezas innatas de cuidado que se fortalecen dentro de los hogares. Y, por otro lado, la retórica de la corresponsabilidad también le ha permitido al Estado deslindarse de su responsabilidad pública en inversión de recursos y capacidades para las personas cuidadoras, argumentando que estos aspectos no constituyen una prioridad, puesto que se gestionan espontáneamente en el entorno privado de las propias familias. Paralelamente, estas han interiorizado este papel, ensamblando argumentos para defender el tipo de cuidado «ideal»y «moralmente superior» que proveen, y para justificar la figura de la mujer cuidadora afectuosa y competente que se genera y forma en sus círculos, a través de la división desigual y sexista de roles entre sus propios miembros.

\section{Conclusiones}

Este estudio exploró las percepciones acerca de la interacción de las políticas destinadas a personas mayores implementadas por el Estado ecuatoriano y la propia agenda de las familias integradas por parientes mayores en la ciudad de Quito, en el camino de su responsabilidad compartida, para velar por los requerimientos de cuidado de este grupo etario. El proceso de realización de entrevistas con las personas responsables del diseño y aplicación de la política social de cuidados para personas mayores en las instancias del Estado, y con los familiares de personas de edad avanzada, permitió constatar la premisa planteada al inicio de este estudio. 
De acuerdo con las vivencias de las personas entrevistadas, el principio de corresponsabilidad entre el Estado y las familias para garantizar los derechos de las personas mayores, señalado en la Constitución del Ecuador y en los planes nacionales de desarrollo establecidos desde el 2009 hasta el 2021, está lejos de cumplirse de forma integral y libre de esencialismos, por cuanto su práctica cotidiana se sostiene en una retórica de un reparto desigual e invariable de los roles que les corresponden a familias y Estado en la organización de los cuidados para personas mayores.

Es posible afirmar lo anterior, dado que, con respecto a la distribución de los papeles en torno al cuidado y aseguramiento de bienestar a la población mayor, se encontraron dos niveles de brechas profundas. La primera se vincula con la noción de una distribución desigual de responsabilidades entre el Estado y de las unidades familiares. La segunda se relaciona con la distribución inequitativa de roles de los trabajos de cuidados entre hombres y mujeres en el interior de los hogares. En otras palabras, en los imaginarios de las personas entrevistadas, se ha asignado automáticamente a los círculos familiares la capacidad natural de la provisión de cuidados, y dentro de este espacio es en las mujeres en quienes se ha repartido la mayor carga del trabajo de cuidado.

Aunque la política social ecuatoriana se ha enfocado en la reducción de la pobreza en la adultez mayor, ofreciendo un piso de seguridad básica y distintas modalidades de atención a las personas mayores de escasos recursos económicos y sin referente familiar, estos esfuerzos no han alcanzado a realizar una mirada holística e interseccional de todas las aristas que constituyen la provisión de bienestar para este grupo etario. Incluir políticas de cuidado en programas de combate a la pobreza no solo legitima la visión de la provisión de cuidado como una respuesta «asistencialista» antes que como un derecho garantizado, sino que, además, normaliza la familiarización de los cuidados. Se asume entonces como una realidad ineludible una función pública débil en la socialización de los cuidados. Esto desemboca en políticas que, al sobreestimar la solidaridad entre generaciones, están centradas en las personas mayores receptoras de los cuidados, al tiempo que niegan e invisibilizan las demandas que surgen desde quienes les proveen cuidados en sus entornos familiares. 
Simultáneamente, la construcción social del cuidado como una actividad propia de las familias ha devenido en la internalización con efectos duraderos de esta percepción en las personas cuidadoras familiares. Es así como en el interior de los hogares se han ensamblado argumentos principalmente relacionados con la obligación moral de cuidar con el horizonte de legitimar, principalmente para las mujeres, la asunción de estas tareas, superando incluso los recursos y capacidades disponibles. Esto con el fin de sortear la sanción social que podría generar «el abandono de las responsabilidades heredadas» de cuidado al pariente mayor.

Por consiguiente, uno de los retos más urgentes para abordar en el análisis de la corresponsabilidad y la distribución de roles entre el ámbito estatal y familiar es ahondar en el análisis de la reciprocidad social (Comas d'Argemir, 2014) no solo como un factor que mueve a las personas a brindar cuidados, sino como un elemento que interviene en cada dimensión de la organización social del cuidado. Por ello es claro que se necesita abandonar la idea de que de la dependencia surge el cuidado como una actividad en la que los «más fuertes» protegen «a los más débiles», para empezar a concebir a la interdependencia como un estado que caracteriza nuestro estar en el mundo y que nos afecta a todos en diferentes contextos y magnitudes. Entonces, si la dependencia es universal e ineludible, no se puede resolver únicamente dentro del espacio privado. Debe convertirse en un asunto de carácter público y, sobre todo, colectivo, que faculte la distribución equitativa, flexible e intercambiable de funciones entre todas las dimensiones de la sociedad. En este punto también deben tomarse en cuenta las agendas políticas en juego, porque a través de estas los Gobiernos, el mercado, las unidades familiares y los grupos comunitarios negocian sus intereses y acceso a recursos.

Finalmente, con miras a apreciar los trabajos de cuidado como una responsabilidad colectiva, es importante también desmantelar los mitos analizados en esta investigación en torno al amor, la abnegación, el sacrificio y la deuda moral que se han incorporado de forma implícita en el entorno familiar y especialmente en los cuerpos femeninos. Justamente son estas generalizaciones las que impiden que las personas que otorgan cuidados puedan combinar sus experiencias como cuidadoras y traba- 
jadoras con independencia de su sexo. Sobre esto, es importante remarcar que la superación de la división binaria y estática de roles entre hombres y mujeres debe venir acompañada de una ardua labor por romper las estructuras injustas de desigualdad y poder propias de un sistema capitalista. Para este son sumamente útiles y favorables las disparidades en la distribución de roles entre hombres y mujeres y la categorización del trabajo doméstico que se les ha asignado a estas últimas como no remunerado, a fin de perpetuar su lógica de acumulación de riqueza y apropiación de la fuerza de trabajo.

Por lo tanto, develar estos estereotipos y mitos sobre el trabajo de cuidado de la familia y, particularmente, de las mujeres, facilitaría que la noción de reciprocidad social en las que se asientan nuestras dependencias pueda extenderse de las fronteras familiares para ubicarse como un asunto público que debe incluirse como uno de los elementos para la garantía integral de bienestar de toda la población en todas sus etapas del ciclo de vida.

\section{Bibliografía}

Acosta, E. (2015). Cuidados en crisis. España: Universidad de Deusto.

Acosta, E., Picasso, F. y Perrotta, V. (2017). Cuidados en la vejez en América Latina. Los casos de Chile, Cuba y Uruguay. Providencia: Fundación Konrad Adenauer.

Acosta, M. (2013). Conciliación con corresponsabilidad: Fortalecienco derechos y generando oportunidades de mejora en el ámbito laboral. PNUD.

Anderson, J. (2007). «Políticas y programas orientados a las familias en los países andinos». En I. Arriaga (ed.). Familias y políticas públicas en América Latina: Una historia de desencuentros. Santiago de Chile: Naciones Unidad, CEPAL.

Aguirre, R. (2007). «Familias como proveedoras de servicios de cuidado.» En A. Irma (ed.). Familias y políticas públicas en América Latina: una historia de desencuentros. Santiago de Chile: Naciones Unidas, CEPAL.

AnTía, F. (2018). «Regímenes de política social en América Latina: una revisión crítica de la literatura». Desafíos, 30 (2), 193-235. 
Aranco, N., Stampini, M., Ibarrarán, P. y Medellín, N. (2018). Panorama de envejecimiento y dependencia en América Latina y el Caribe. Banco Interamericano de Desarrollo.

Arriagada, I. (2010). «La crisis de cuidado en Chile». Revista de Ciencias Sociales, (27), 58-67.

Asamblea Constituyente del Ecuador (20 de octubre de 2008). Constitución de la República del Ecuador. Disponible en:<http://www.asambleanacional.gob.ec/sites/default/files/documents/old/constitucion_de_bolsillo.pdf>.

Asamblea Nacional del Ecuador (2010). Ley Orgánica de Servicio Público. Quito.

Ascensio, M. S. (2017). «Regímenes de bienestar y políticas de género en América Latina. Una nueva relación entre Estado, mercado, comunidades y familias». Rumbos TS, (16), 179-192.

BATTHYÁNy, K. (2015). Las políticas y el cuidado en América Latina. Montevideo: CEPAL.

BENERÍA, L. (2007). «The crisis of care, international migration and the capabilities approach: Implications for policy». En D. H. al. (ed.). Mario Einaudi: Center for International Studies (Woorking Paper Series), 1-21

Carbonell, M., Gálvez, L. y Rodríguez, P. (2014). «Género y cuidados: respuestas sociales e institucionales al surgimiento de la sociedad de mercado en el contexto europeo». AREAS. Revista Internacional de Ciencias Sociales, (33), 17-32.

Carmona, V., Siavil, C. y Ribot de Flores, S. (2007).«Implicaciones conceptuales y metodológicas en la aplicación de la entrevista en profundidad». Laurus: Revista de Educación, 13 (23), 249-262.

Carrasco, C. Borderías, C. y Torns, T. (2019). «Introducción. El trabajo de cuidados: antecedentes históricos y debates actuales». En C. CArrasCO, C. Borderías y T. Torns (eds.). El trabajo de cuidados. Historia, teoría y políticas. Madrid: FUHEM Ecosocial-Los libros de la Catarata.

Cerri, C. y Alamillo-Martínez, L. (2012). «La organización de los cuidados, más allá de la dicotomía entre esfera pública y esfera privada». Gazeta de Antropología, 28 (2), 1-23. 
Comas d’Argemir, D. (2014). «Los cuidados y sus máscaras. Retos para la antropología feminista». Mora, (20), 167-182.

Comas d’Argemir, D. y Roca, J. (1996). «El cuidado y asistenciacomo ámbito de expresión de la tensión entre biología y cultura». En D. ConTreras (ed.). Reciprocidad, cooperación y organización comunal. Zaragoza.

Consejo Nacional de la Niñez y la Adolescencia (2013). Agenda Nacional para la Igualdad Intergeneracional 2013-2017. Quito.

D’Alessandro, M. (2018). Economía feminista. Las mujeres, el trabajo y el amor. Buenos Aires: Penguim Random House.

DALY, M. y LEWIS, J. (2000). "The concept of social care and the analysis of contemporary welfare states». British Journal of Sociology, 51 (2), 281298.

Del Valle, A. (2013). «Bienestar, familia y problemas de cuidados en América Latina». Ponta Grossa, 13 (2), 27-45.

Del VAlle, T. (2010). «La articulación del parentesco y el género desde la antropología feminista». En V. Fons, A. Piella y M. Valdés. Procreación, crianza y género. Aproximaciones antropológicas a la parentalidad. Barcelona: Promociones y Publicaciones Universitarias.

Eguren, N. (2001). «Familia y Estado: el reparto del bienestar». Abaco: Revista de cultura y ciencias sociales, 29 (30), 101-112.

EsPing-ANdersen, G. (2000). Fundamentos sociales de las economías postindustriales. Barcelona: Ariel.

Esquivel, V. (2011). La economía del cuidado en América Latina: Poniendo a los cuidados en el centro de la agenda. El Salvador: Programa de las Naciones Unidas para el Desarrollo.

Esteban, M. (2011). Crítica del pensamiento amoroso. Barcelona: Bellaterra.

FEDERICI, S. (2018). Revolución en punto cero: Trabajo doméstico, reproducción y luchas feministas. Madrid: Traficantes de sueños.

García, B. y De Oliveira, O. (2011). «Cambios familiares y políticas públicas en América Latina». Annual Review of Sociology, 37, 613-633.

Gherardi, N., Pautassi, L. y Zibecchi, C. (2012). De eso no se habla: el cuidado en la agenda pública. Estudio de opinión sobre la organización del cuidado. Buenos Aires: Equipo Latinoamericano de Justicia y Género. 
Gómez, E. (2008). «La valoración del trabajo no remunerado: una estrategia clave para la política de igualdad de género». En Organización Panamericana de la SAlud (ed.). La economía invisible y las desigualdad de género. La importancia de medir y valorar el trabajo no remunerado. Washington.

GonzÁlveZ, H. (2016). «Los cuidados en la migración transnacional». Sur24, 13 (24), 43-52.

Huenchuan, S. y Guzmán, J. (2007). «Políticas hacia las familias con personas mayores: el desafío del cuidado en la edad avanzada». En I. ArRIAGA (ed.). Familias y políticas públicas en América Latina. Una historia de desencuentros. Santiago de Chile: Naciones Unidas, CEPAL.

IZQUIERDO, M. J. (2004). «Del sexismo y la mercantilización del cuidado a su socialización: Hacia una política democrática del cuidado». En Fondo Social Europeo e Instituto Vasco de la Mujer (eds.), Congreso Internacional Sare 2003: Cuidar cuesta: costes y beneficios del cuidado. Vitoria-Gasteiz: EMAKUNDE-Instituto Vasco de la Mujer.

LEWIs, J. (2019). «Género, envejecimiento y el "nuevo pacto social": la importancia de desarrollar un nuevo enfoque holístico de las políticas de cuidados». En C. CARrasco, C. Borderías y T. Torns (eds.). El trabajo de cuidados. Historia, teoría y políticas. Madrid: FUHEM Ecosocial-Los libros de la Catarata.

Marco, F. y Rodríguez, C. (2010). «Pasos hacia un marco conceptual sobre el cuidado». En S. Montaño y C. CALDerón. El cuidado en acción. Entre el derecho y el trabajo. Santiago de Chile: Organización de las Naciones Unidas.

Martínez, J. (2007). Regímenes de bienestar en América Latina. Madrid: Fundación Carolina.

- (2008). ¿Arañando bienestar? Trabajo remunerado, protección social y familias en América Central. Buenos Aires: CLACSO.

Martínez, J. y Voorend, K. (2009). Sistemas de patriarcado y regímenes de bienestar en América Latina. Madrid: Fundación Carolina.

Ministerio de Inclusión EConómica y Social (15 de octubre de 2016). Diseño de una política pública a favor de los derechos de las personas adultas mayores. Disponible en: <http://www.inclusion.gob.ec/category/el-ministerio/>. 
Ministerio de Inclusión Económica y Social (22 de mayo de 2020). Dirección Población Adulta Mayor. Disponible en: <https://www.inclusion.gob.ec/ direccion-poblacion-adulta-mayor/>.

Minteguiaga, A. y Ubasart-González, G. (2013). Revolución ciudadana y régimen de bienestar en Ecuador (2007-2012). Buenos Aires: Consejo Latinoamerica de Ciencias Sociales.

Nieves, M. y Robles, C. (2017). El cuidado, pilar de la protección social: derechos, políticas e institucionalidad en América Latina. En CEPAL (ed.). Institucionalidad social en América Latina y el Caribe. Santiago de Chile: CEPAL.

O'Connor, J. (1996). «From women in the welfare state to gendering welfare state regimes». Current Sociology, 44 (2), 1-130.

Orloff, A. (1993). "Gender and the social rights of citizenship: The comparative analysis of gender relations and welfare states». American Sociological Review, 58 (3), 303-328.

PÉRez, A. y López, S. (2011). Desigualdades a flor de piel: cadenas globales de cuidados. Concreciones en el empleo de hogar y politicas públicas. Madrid: ONU Mujeres.

RAZAVI, S. (2007). The political and socialeconomy of care in a development context. Geneva: United Nations Research Institute for Social Development. Rico, M. N. (2016). Políticas de cuidado en América Latina: Forjando la igualdad. CEPAL, Naciones Unidas.

Rodríguez, C. (2015). «Economía feminista y economía del cuidado: aportes conceptuales para el estudio de la desigualdad». Nueva Sociedad, 256, 30-44.

Rossel, C. (2013). Políticas para las familias en América Latina: Panorama de politicas de reducción de pobreza y conciliación. Buenos Aires: UNESCO.

Secretaría General de Comunicación de la Presidencia (3 de abril de 2018). « ¡Sin paquetazos, sin afectar a los más pobres!», Presidente de la República presentó el Programa Económico. Disponible en: <https://www. comunicacion.gob.ec/sin-paquetazos-sin-afectar-a-los-mas-pobrespresidente-de-la-republica-presento-el-programa-economico/>.

Secretaría Nacional de Planificación y Desarrollo (2009). Plan Nacional para el Buen Vivir 2009-2013. Quito. 
Secretaría Nacional de Planificación y Desarrollo (2013). Plan Nacional para el Buen Vivir 2013-2017. Quito.

- (2017). Plan Nacional de Desarrollo 2017-2021. Toda una vida. Quito.

Vega, C., Martínez, R. y Paredes, M. (2018). «Introducción. Experiencias, ámbitos y vínculos cooperativos en el sostenimiento de la vida». En C. Vega, R. Martínez y M. Paredes (eds.). Experiencias y vínculos cooperativos en el sostenimiento de la vida en América Latina y sur de Europa. Madrid: Traficantes de sueños.

Wood, G. y Gough, I. (2006). A comparative welfare regime approach to global social policy. World Development, 34 (10), 1696-1712. 